\title{
The Benefits and Challenges of Large Scale MVILV Distribution Network Modelling: A Case Study
}

This paper was downloaded from TechRxiv (https://www.techrxiv.org).

\section{LICENSE}

CC BY-NC-SA 4.0

SUBMISSION DATE / POSTED DATE

18-02-2022 / 23-02-2022

\section{CITATION}

Mehigan, Laura; Zehir, Mustafa Alparslan; Cuenca, Juan; Sengor, Ibrahim; Geaney, Ciaran; Hayes, Barry (2022): The Benefits and Challenges of Large Scale MV/LV Distribution Network Modelling: A Case Study. TechRxiv. Preprint. https://doi.org/10.36227/techrxiv.19196246.v1

$\mathrm{DOI}$

10.36227/techrxiv.19196246.v1 


\title{
The Benefits and Challenges of Large Scale MV/LV Distribution Network Modelling: A Case Study
}

\author{
Laura Mehigan, Graduate Student Member, IEEE, Mustafa Alparslan Zehir, Member, IEEE, \\ Juan J. Cuenca, Graduate Student Member, IEEE, Ibrahim Sengor, Member, IEEE, \\ Ciaran Geaney, Member, IEEE, Barry P. Hayes, Senior Member, IEEE
}

\begin{abstract}
Power distribution networks are experiencing large deployment of behind-the-meter distributed generation and storage along with electrified transport and heat, requiring fundamental changes in planning and operation. Synthetic representative networks may not be sufficient to fully address the needs of researchers, operators and decision-makers. There are increasing efforts to directly model large-scale real networks and conduct detailed analysis beyond conventional practices. This paper presents a large-scale medium voltage (MV)-low voltage $(\mathrm{LV})$ integrated network model building, scenario determination and analysis approach for distribution networks. Data with different formats from several databases were used in model building. The model is validated using benchmark scenarios based on historical network measurements from the field. In collaboration with the national distribution system operator in Ireland (ESB Networks), a pilot study was conducted for a rural network in the Southwest of Ireland, highlighting possible challenges and offering suitable solutions for country-scale implementation.
\end{abstract}

Index Terms-power distribution, distribution control, distribution lines, distribution planning, power system simulation.

\section{INTRODUCTION}

D ISTRIBUTION grids are on the frontline of bottom-up transformation of power systems [1]. Efforts towards decarbonization, decentralization, integration and digitalization are shaping the necessities for network planning and operation [2]. 2019 was a record-breaking year for electric vehicles (EVs), as annual sales were around 2 million and the global stock reached 5.1 million due to annual market growth rates in excess of 50\% in the last decade [3]. In 2020, despite pandemic challenges the market has grown by $41 \%$ with record-breaking

This work was supported in part by the Collaborative Research of Decentralization, Electrification, Communications and Economics (CREDENCE) Project, funded by Science Foundation Ireland under Grant 16/US-C2C/3290.

L. Mehigan is with the MaREI Centre, Energy Policy and Modelling Group, Environmental Research Institute, University College Cork, Cork T23 XE10, Ireland (e-mail: laura.mehigan@ucc.ie).

M. A. Zehir is with the Department of Electrical and Electronics Engineering, Faculty of Engineering, Marmara University, Istanbul 34840, Turkey (e-mail: alparslan.zehir@marmara.edu.tr).

J. J. Cuenca is with the School of Engineering and Architecture, University College Cork, Cork T12 K8AF, Ireland (e-mail: j.cuenca@umail.ucc.ie).

I. Sengor is with the MaREI Centre, Environmental Research Institute, University College Cork, Cork T23 XE10, Ireland and the Department of Electrical and Electronics Engineering, Izmir Katip Celebi University, İzmir 35620, Turkey (e-mail: isengor@ucc.ie).

C. Geaney is with ESB Networks, Leopardstown Road, Dublin 18, D18 XN80, Ireland (e-mail: ciaran.geaney@esb.ie).

B. P. Hayes is with the MaREI Centre, Environmental Research Institute, University College Cork, Cork T23 XE10, and the School of Engineering and Architecture, University College Cork, Cork T12 K8AF, Ireland (e-mail: barry.hayes@ucc.ie). annual sales, exceeding 3 million. In 2021 with arising supply challenges in the industry, annual electric car sales more than doubled to 6.6 million, reaching around $9 \%$ of the global car market and more than tripling market share compared to 2019 [4]. From 2019 to 2024, residential, commercial and offgrid distributed photovoltaic (PV) installations are expected to more than double, constituting nearly half (more than 300 GW) of the overall solar PV capacity growth [5]. At low penetration levels of new technologies, while transmission system operators (TSO) do not face considerable problems, distribution system operators (DSO) observe bus voltage and line loading limit violations which become more significant at large penetration rates, especially in rural grids [6].

There is a significantly more assets in distribution grids compared to transmission grids. In Ireland, there are over 20 times more lines, 500 times more substations and 3700 times more transformers at the distribution level compared to the transmission level [7]. This structural complexity meant that distribution networks were conventionally modelled and analyzed considering mainly the primary or MV systems, while the secondary or LV sides of the networks have limited details or are not even included [8]. Planning is conventionally done according to foreseen annual peaks, usually considering a linear demand growth rate specifically determined for the considered territory. For urban areas, there are more sophisticated different growth rates, such as the industrial areas in the city. In touristic areas of a country, growth rates can be very different, while in rural areas it is much flatter. The preliminary approach to handle the initial integration of EV chargers, batteries, electric heating and other new assets is to have a reinforceable system and follow a wait and see approach. Only for large facilities, there is an initial application procedure and a lead planning time to respond to such major changes in the operated network. On the other hand, locally concentrated behind-the-meter integration of new assets (such as installing electric heaters in large residential complexes, special offers in setting up domestic EV chargers and other) may accelerate the escalation of highly localized network issues at the LV level. It may be more challenging for the system operator to deal with such problems, as reinforcements on the main feeder and transformer may not satisfactorily address highly localized network issues.

Increasing integration of distributed energy resources on the secondary or LV side of distribution transformers led to three major trends in network analysis: benchmark LV networks, clustered representative LV feeders and large-scale LV models 
integrating real system data. Benchmark networks are primarily used for initial exploration of low-carbon technologies (LCT) integration and related concepts in example grids; but they usually do not represent the diverse characteristics of different networks. Selected or designed feeders representing the topological features of different clusters considerably reduce the number of networks and analysis efforts in region- or country-wide impact analysis studies. On the other hand, highlighting the exact performance, limitations and benefits in each LV area may require more detailed and larger scale models that can be built through direct integration of real system data. Moreover, the aggregated impacts of several assets from different LV areas on the MV level can be explored through MVLV integrated simulations. Scenario determination and analysis are some other important areas requiring improvements in distribution network planning and operation. Deterministic snapshot simulation of the worst cases or extreme scenarios is increasingly replaced with time series simulation of diverse supply and demand profiles. This emerging trend can provide further insights into the potential impacts such as the occurrence rate, duration, severity/magnitude, and times/periods of possible issues/flexibilities that may be faced/achieved through integration of the explored technologies and concepts even covering seasonal differences [9].

This paper presents an MV-LV model building, automated scenario determination and analysis approach for distribution grids. Large scale detailed network models were built using real data with diverse formats from different databases. The developed models were validated by first considering each LV area individually, then collectively including the MV level. Time series simulations were explored in comparison with the conventional analysis scenarios, examining the added value of more detailed analysis. A pilot study was carried out for a selected area in the Dingle Peninsula, Ireland to highlight the possible challenges and provide suitable solutions for countryscale implementation.

The main contributions are:

1) To conduct integrated penetration analysis of $P V, E V$ and heat pumps (HP) using realistic models,

2) To identify the challenges of developing large scale integrated MV-LV models for DSO,

3) To analyze the benefits of conducting integrated penetration analysis for DSO.

Section II of the paper provides an overview of the latest state-of-the-art and identifies the gaps that should be addressed. Section III presents the methodologies developed throughout the course of this work. Section IV is devoted to the pilot study details and its results. Section V concludes the paper by discussing the findings and providing directions for future research.

\section{StATE OF The ART}

This section presents the main developments in the areas of distribution network modelling and analysis, identifying the emerging trends and emphasizing the current gaps.

In terms of model development, a study identifies four approaches that best generalizes the prominent works in the literature: manual design, clustering and combining, use of real data through feeder anonymization and automated planning tools [10]. Manual design and clustering are used for synthetic network modelling, while feeder anonymization is for modelling networks based on real data and automated planning can serve both purposes. Benchmark LV networks are systems that are developed for running preliminary studies on grid integration of new technologies, validating new analysis and control algorithms. Benchmark systems can be categorized as feeder type networks and large scale networks [11]. Some networks comprise a substation with several different feeders such as residential, commercial and industrial feeders as in [12] or local generation and storage assets to represent a microgrid case as in [13]. These studies aim to provide a system available for use to researchers, rather than presenting a replicable model building approach, as they develop the models in a straightforward and deterministic way. There are also some replicable approaches such as Reference Network Model greenfield approach using the general statistics provided by DSO [14] or clustering several networks based on k-means together with Euclidean distance [15], fuzzy k-medoids [16] or a combination of several methods (k-meoids++, k-means++, Gaussian Mixture Model together) [17]. The resulting networks can represent diverse topologies and characteristics up to an extent, with tolerable differences in analysis results of the representative ones and the rest of the networks in the identified clusters. Production of synthetic models may require some assumptions for simplification, such as equal distribution of branches on a main feeder, consecutive distribution of load points and other. Statistical, operational and expert validation approaches are suggested to evaluate the realism of synthetic networks [18].

A study that reviewed the distribution test feeders, identified the main limitations as,

- small size,

- lack of time series data,

- lack of representativeness,

- missing geographical coordinates,

- design and data availability for a single purpose and isolated feeders,

specifically emphasizing the need for building larger-scale networks [19].

Another comprehensive review study states that synthetic networks may not be sufficiently representative and may fail to replicate real grid structures comprehensively due to diverse properties of real networks [13]. A recent alternative and hybrid approach is to use a mixture of real network data from different parts of the network to develop large scale integrated MV-LV models [20]. Although the considered networks are not directly connected to each other in reality, the developed integrated model can serve as a highly realistic model for researchers. In a similar manner, direct modeling of large scale real MV-LV networks is a recent approach that gains particular interest [21]. There are efforts to automate the determination and exploration of the desired planning and operational scenarios. Load allocation to customer nodes can be organized to match feeder level real measurements as in [22]. Smart 
meter data can be automatically allocated to customer nodes using Euclidean distance as in [23]. A recent study proposes the use of advanced metering infrastructure (AMI) data from customer nodes, together with data acquisition system (DAS) measurements at MV-to-LV transformers to determine timeseries load profiles, comprising the unmetered loads [24]. Building large scale networks models by direct integration of real data from the field can also enable real-time analysis and digital twin applications. A conceptual explanation of real-time distribution network analysis is available in [25] and a pilot application examining its applicability in the field is presented in [26]. In terms of analysis, the approach taken depends on the purpose of the study. Quasi-Static Time Series (QSTS) analysis is among the four main approaches mentioned in the draft IEEE guide on conducting DER impact studies investigating equipment control, operation and voltage regulation. The other approaches are dynamic simulation for stability and voltage frequency ride-through studies, electromagnetic transient simulation for protection design and fault analysis and harmonic and flicker study for power quality of feeders [27]. Unlike snapshot simulation, QSTS allows accurate analysis of DER impacts on voltage variations, energy and loss calculations closer to daily operational cases, preventing overestimation of impacts due to sole consideration of extreme peaks. It can provide accurate quantification of magnitude, occurrence and duration of impact.

This study contributes to the literature by developing largescale integrated MV-LV network model building, automated scenario determination and advanced analysis approach using real network and customer data from diverse databases. Tool independent modeling and analysis approaches are developed that could be replicated to develop integrated MV-LV models across the entire country grid.

\section{Methodology}

This section explains the methodology used for developing the integrated MV-LV model. As seen in Fig. 1, an MV model used for DSO network planning purposes of a particular area is converted from commercial planning software format to the open source OpenDSS format. The OpenDSS MV model is validated against the original DSO network planning model to confirm that the voltage profile obtained with the OpenDSS MV model is reasonable. In parallel, data is extracted from the DSO geographic information system (GIS), asset management and operational databases for the individual LV areas, located within a subset of the geographical area covered by the MV model. Using tool independent data management, missing/incorrect data is auto-detected and corrected. Subsequently, OpenDSS models for the individual LV areas are created. Each LV model is validated using historical information, where available. Integration of the MV and LV models then takes place, taking care to ensure alignment of the respective model geometries. The integrated MV-LV model is then validated, using historical measurements and through the assessment made in consultation with the DSO operator for the modelled area.Scenarios are determined for PV, EV, and HP penetrations and comparative analyses performed to determine the benefit of detailed LV modelling as indicated in Fig. 1.

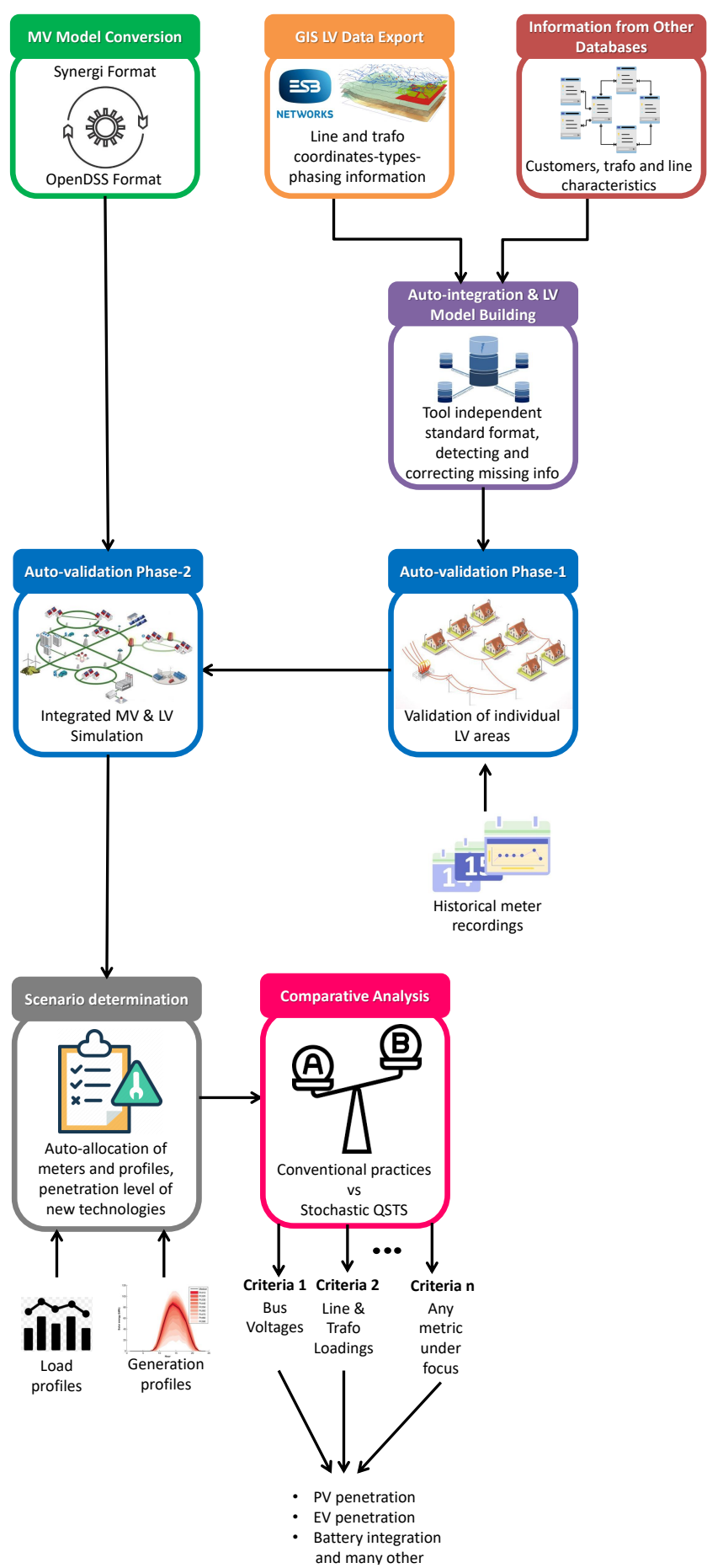

Fig. 1: Overview of modelling process

\section{CASE Study}

\section{A. The Modelled Network}

A real $10 \mathrm{kV}$ MV feeder owned and operated by the Irish DSO ESB Networks, with 2,188 customers is used for this work. The network comprises a mixture of underground and overhead conductors. The customers are predominantly residential with a significantly smaller number of industrial/commercial customers. In addition to the MV feeder, a total of 51 individual real LV networks are modelled connect- 


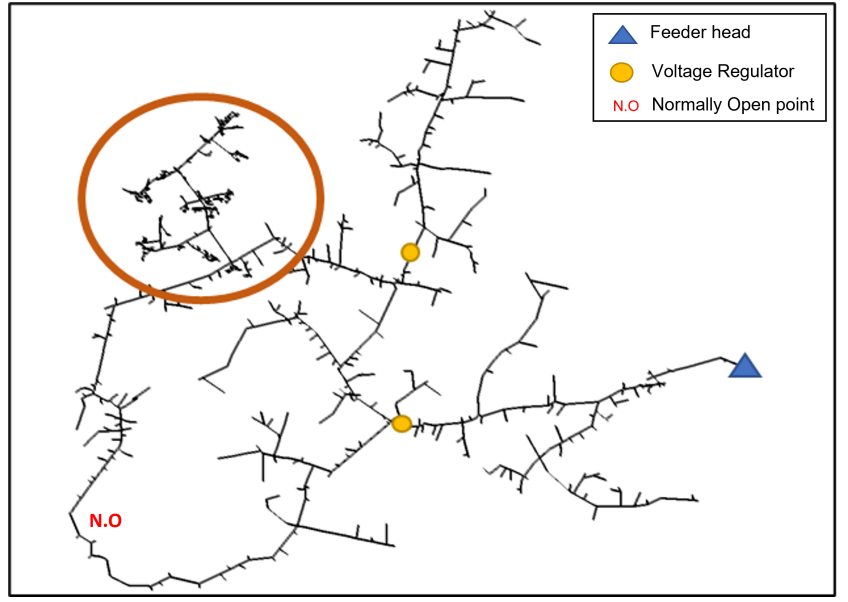

Fig. 2: Integrated MV-LV modelled area (circled area is the detailed LV section).

ing 317 customers, inclusive of 123 -phase customers. The remaining 1,871 customers are represented by loads connected directly to the MV network. The number of customers per LV network varies from 1 to 56 reflecting the reality of the mixture of one-off housing and holiday home estates in many coastal areas in Ireland. The distance of customers to the MV/LV transformer in the LV networks also varies significantly, the nearest is $\sim 18 \mathrm{~m}$, the furthest $\sim 370 \mathrm{~m}$ and the average distance of customers within all $51 \mathrm{LV}$ networks is $\sim 149 \mathrm{~m}$. The 51 MV/LV transformers range in size from 5 to 200kVA. The $38 \mathrm{kV}$ substation at the head of the feeder supplies the $10 \mathrm{kV}$ feeder (and other $20 \mathrm{kV}$ feeders not included in this model) via a mix of both $38 / 10 \mathrm{kV}$ and $38 / 20 \mathrm{kV}$ transformers. The $10 \mathrm{kV}$ side of this feeding substation is represented by the blue triangle in Fig. 2, i.e., the feeder head for the purpose of this model. The feeder voltage is set to $1.05 \mathrm{pu}$ in line with typical network operation by the DSO for this substation.

\section{B. Load Allocation and Determination of PV, EV, and HP profiles}

Load allocation was carried out separately for the LV and MV parts of the network. Within the modelled LV network, load allocation was confined to line end points starting with the furthest point from the MV-LV transformer and divided equally among the endpoints. Loughborough University's CREST Demand Model [28] was used to generate stochastic individual daily load profiles for each residential customer in a manner that ensured the measured transformer peak demand at each of the $51 \mathrm{MV} / \mathrm{LV}$ substations did not exceed operational tolerances allowed under the DSO security and planning standards [29]. CREST Demand Model is an opensource tool that has already been cited and used in over a thousand studies. It is based on household usage statistics of the United Kingdom, meteorological data and appliance parameters. It provides the specification of the number of people, the day of the year and assignment of appliances in a dwelling. Following a bottom-up approach and using stochastic programming methods, it aggregates the consumption behavior of each appliance and produces residential load

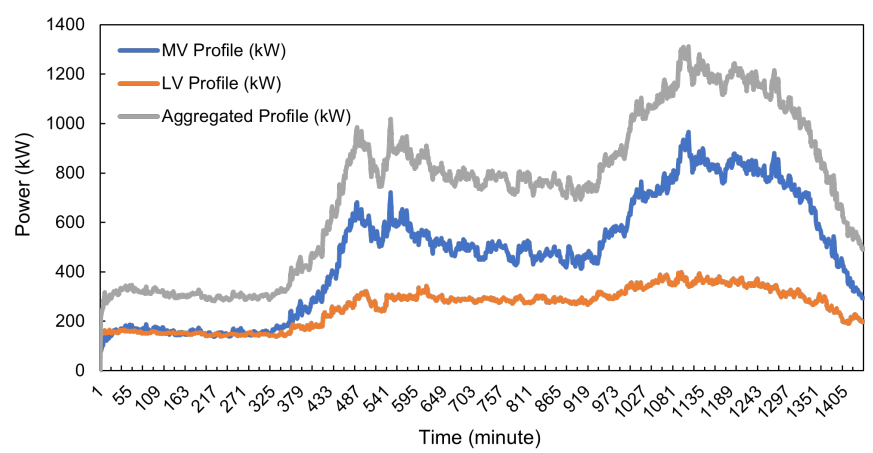

Fig. 3: 24 hour base load profile for MV, LV and combined MV and LV demand

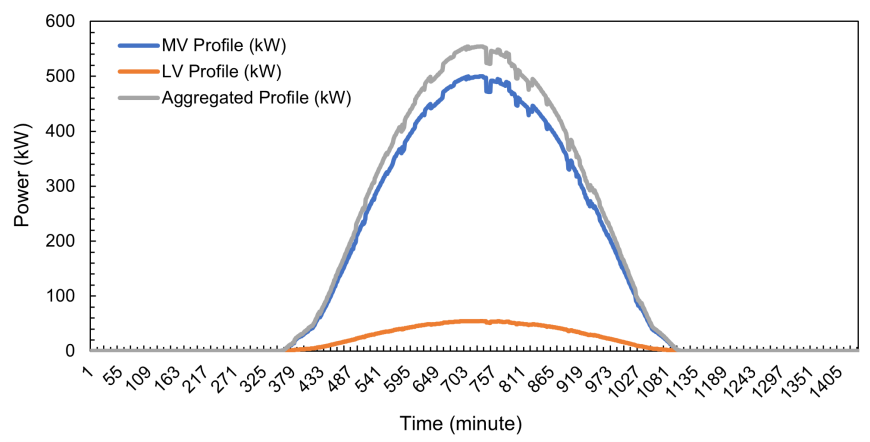

Fig. 4: Daily aggregated PV production profile for MV, LV and combined MV and LV with $15 \%$ penetration level

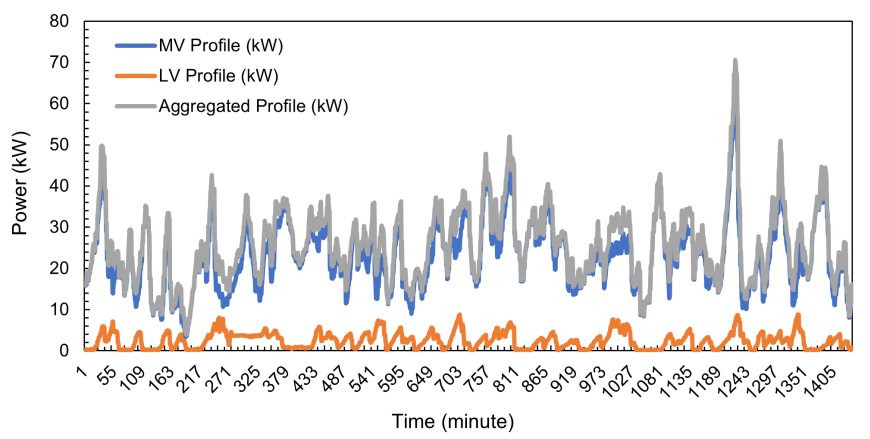

Fig. 5: Daily aggregated HP demand profile for MV, LV and combined MV and LV with $15 \%$ penetration level

profile for a day with 1 min resolution. The details of the tool together with its performance verification can be found in [28].

Synthetic load profiles using the annual total energy consumption data were generated for each commercial customer using the commercial load hourly average per unit values presented in previous literature [30]. For the remainder of the MV network modelled, where LV is not specifically modelled, bulk MV loads to represent the remaining 1,871 customers were created using synthetic load profiles based on the peak load and number of customers connected to each MV point. The generated load profiles for MV, LV, together with combination of MV and LV are depicted in Fig. 3.

For PV, a standard installation size of $2.1 \mathrm{kWp}$ was considered. This reflects the size adopted in a recent pilot project by the DSO in the area modelled [31]. The percentage penetration of $\mathrm{PV}$ is attributed to the percentage of the number of customers in each substation and it is rounded up to the nearest 


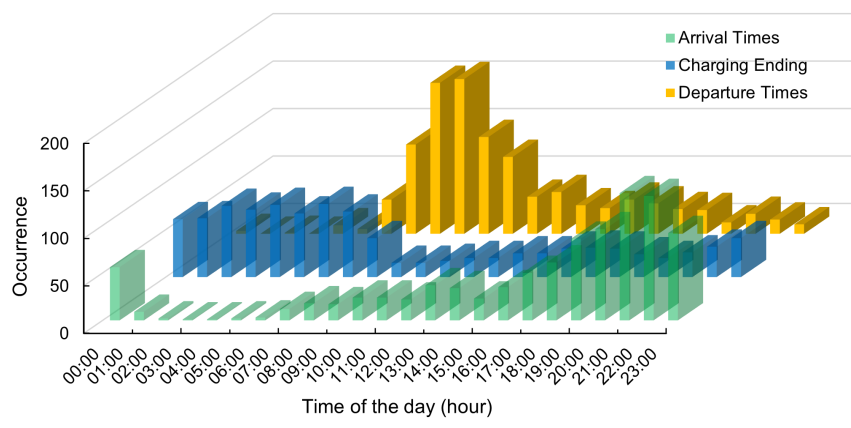

Fig. 6: EV arrival, departure and charging starting times recorded during the field pilot

integer. In addition, it is assumed that each customer is able to have a $2.1 \mathrm{kWp}$ standard PV size. For example, in the case of $10 \% \mathrm{PV}$ penetration, a substation with 48 customers, the 5 furthest away customers are going to have $2.1 \mathrm{kWp} \mathrm{PV}$ installed (i.e., $10 \%$ X 48 equates to $4.8 \approx 5$ customers). PV profiles were derived using another version of the CREST model, which can generate stochastic solar irradiance profiles with 1-min resolution, based on meteorological statistics, including cloudiness [32]. Figure 4 depicts the aggregated PV production profiles for only LV customers, only MV customers, and a combination of them in case of $15 \%$ penetration level.

The data from the DSO project [31] into EV use and smart charging in the area modelled was used to generate statistics for EV arrival/charging start time, charging end time, and EV departure time as demonstrated in Fig. 6. This enabled the generation of $951 \mathrm{EV}$ charging profiles with 1 min resolution. In line with the field pilot, charging power is considered as $7.4 \mathrm{~kW}$. Similar to the findings of the pilot study, EV charging typically takes place between $11 \mathrm{pm}$ and $8 \mathrm{am}$ taking advantage of the reduced night-time tariff rate. Combinations of the EV profiles are randomly selected and assigned within the network to reflect the relevant percentage of EV penetration under assessment. Similarly, HP profiles were modelled based on measurements taken from energy meters installed on the $5 \mathrm{HPs}$ installed as part of the DSO project. Figure 5 demonstrates the aggregated HP power demand profiles for only LV customers, only MV customers, and a combination of them in case of $15 \%$ penetration level. Scenarios involving PV, EV, and HP separately were investigated, as well as every technology combination. Increases of $1 \%$ penetration were simulated until the model ceased to converge. This non-convergence is explained by the fact that for every technology case, the maximum penetration level achieved is considerably larger than the peak loading conditions of the feeder, i.e., the feeder is not designed to handle larger currents, power flows, voltage drops and rises, etc.

\section{Challenges of Large Scale integrated MV-LV model devel- opment}

A key focus of this work, undertaken in collaboration with the Distribution System Operator (DSO) in Ireland, was repeatability as the same methodology may be used for modelling additional parts of the distribution network in the future. A number of challenges were encountered during the model development process. These challenges encountered can be grouped into a number of broad categories:

1) Legacy asset data issues: Within power utilities there is often a diverse range of data sources and databases relating to network assets such as GIS, Asset Management Systems (AMS) and Energy Management Systems (EMS) [33]. While 45 years is considered the typical length for distribution network asset life from a business perspective in Ireland [34], the technical reality is that many assets remain fully functional and operational on the network beyond this timeframe, particularly at lower voltage levels. Records for such older network assets, commissioned at a time when paper records were standard, were often stored in local area offices rather than a central repository with cataloguing and archiving approaches varying from area to area. A backup of digital records is commonplace nowadays, however backups of paper records were not standard in the past. Thus, even though digital records of every asset typically exist today, the level of detail contained in these records for older assets is dependent on the existence and condition of the paper record at the time the digital records of the asset were created. This issue is often the cause of missing asset data which can make it difficult to create models of real networks.

2) Difference in geometries between $M V$ and $L V$ network: The geometry of an asset is used to locate it on a map and this works very well for significant lengths of line and cable assets. Other assets, although essential to the network and documented in the asset registry for the distribution network, may not need to be mapped due to their small lengths. Thus, they may not be specified in a GIS system. These include assets such as jumpers and lead-ins. This can lead to a misalignment in geometries when developing $\mathrm{MV}$ and LV network models. Additionally, if the origin of geospatial information is not the same for the MV network and the LV network this can introduce further differences in the alignment of the model geometries. This is particularly a problem when it comes to integration of MV and LV models.

3) Timing of $\boldsymbol{M V}$ and $\boldsymbol{L V}$ data extraction: By their nature distribution networks are constantly evolving, with network upgrades and reinforcements, phasing changes to address unbalance issues, and new connections. By availing of already existing MV models, as was adopted in the methodology in this paper, there can be a difference in the date at which data was extracted to build the MV and LV models, leading to higher chance that the MV model does not fully reflect the real MV network at the time the LV models were built. Updating the MV network can be a labour intensive process and if it is not completed, it can cause problems at model integration stage, particularly if phasing is not correct.

4) Availability of historical data: To validate an integrated MV-LV model, ideally a full set of historical network and customer meter data would be available. In reality, this is challenging. For this work, real historical metering data for individual customers was available from the DSO project, where permission was sought and obtained from each individual customer to allow recording of meter data for a specific period only. Typically, individual customer metering data is not available due to privacy and regulatory issues. Aggregated 
customer data can be used but would typically be located at the MV-LV transformer point. Due to the radial nature of the distribution network and its historical unidirectional power flow paradigm, real time network monitoring of $\mathrm{MV} / \mathrm{LV}$ is not widespread. Although, it may be warranted with the increase in distributed and behind-the-meter generation, there can be challenges in acquiring this data remotely, particularly in rural areas with poor telecommunications coverage. This makes model validation particularly challenging.

\section{Benefits of integrated MV-LV modelling}

The fundamental role of a DSO is to operate a safe secure and reliable network in as economic a manner as possible. The traditional approach using design rules of thumb had significant merit. However, the increasing volume of DER at $\mathrm{MV}$ and LV is changing the traditional unidirectional power flow of radial distribution networks. Integrated modelling of MV-LV networks provides an opportunity for DSO to ascertain whether the enduring use of these design principles sufficiently meet the requirements of the fundamental role of a DSO. Integrated MV-LV models offer several additional benefits for DSO and the research community including the ability to:

1) Identify strain on the LV circuits due to local energy balancing

2) Evaluate suitable network reinforcements to harness the benefit of combinations of DER technologies and minimize cost

3) Quantify the impact of network investment on network losses

4) Estimate hosting capacity across the network

5) Assess the impact and efficacy of proposed operational strategies to address network issues such as active voltage regulation, phase changes, curtailment or other.

6) Provide a base model for developing detailed models required for dynamic stability or harmonics studies.

While there are challenges to developing these models in the first instance, once developed the overhead in updating and modifying existing elements of the models is relatively small, particularly if a suitable naming convention has been employed. Furthermore, the range of scenarios and technology types that can be explored is extensive, particularly when using an open source modelling tool such as EPRI OpenDSS. Such models, complemented by suitably located monitoring devices could also be used in the development of state estimation tools for real time network monitoring by DSO.

\section{Results}

Numerous simulations have been conducted to investigate the impacts integrating new technologies or their combinations thereof on the MV/LV distribution network in the Dingle peninsula. It should be stated that all the obtained results could not be presented in the paper due to page limitations and the sake of clarity.

All measurements are monitored at three different points in the model that are, in turn, primary sides of LV transformers, secondary sides of transformers, and furthest customers in the related substation. A boxplot for voltage levels measured at

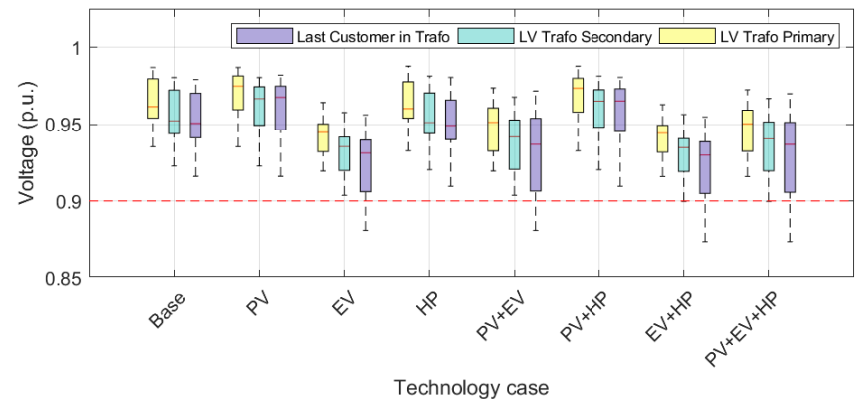

Fig. 7: Voltage measured at different points for a selected transformer (ID 13) for each case

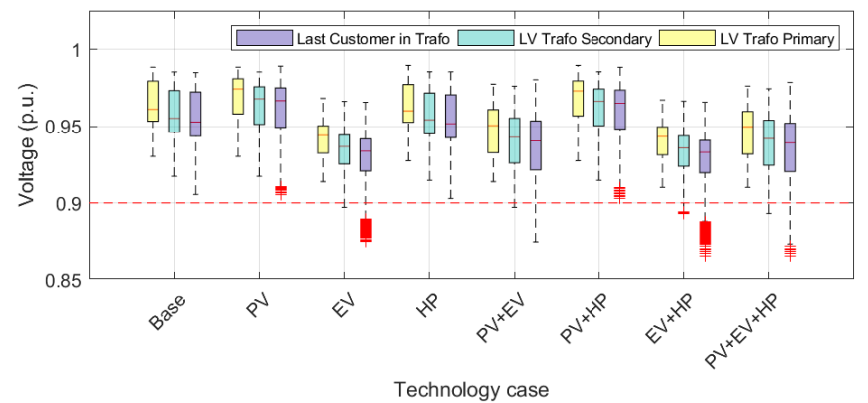

Fig. 8: Voltage measured at different points for all transformers and each case

different points for a transformer (ID 13) is demonstrated in Fig. 7 in case of both individual penetrations and combinations of the evaluated technologies. It is worth underlining that the penetration level is $15 \%$ here for each technology and the transformer ID 13 has been given as an example here due to remarkable changes in voltage levels. As can be concluded from the mentioned figure, while only PV penetration causes voltage rise, it is still within the limits, EV penetration for both individual penetration and its combinations with other technologies (dual and triple combinations) results in relatively lower voltages. In addition, voltage exceeding the lower bound limits can be seen in each case which is including EV integration.

Comparative analysis results of LV transformer primary, secondary and the furthest LV customer for all transformers and each technology integration scenarios is shown in the Fig. 8 and 9. Results for each LV area are provided in the Appendix. PV integration results in increased average and highest voltage values, with no considerable improvement in the lowest voltages observed in daily operation (see Fig. 10b). This is mainly due to occurrence of daily peak demand in the evening, when there is no output from PVs. Integration of HPs has minor impacts on the highest, average, and lowest observed voltages, since they usually simultaneously operate early in the morning, during low demand times (see Fig. 10c). Integration of EVs, considerably reduces the highest, average, and lowest observed voltage values, triggering undervoltage issues at customer nodes at the same penetration level (see Fig. 10d). This is due to high charging rates of chargers and the coincidence of simultaneous EV charging with high residential demand times.

Combined integration of PV with EV increases the average and the highest observed voltages (see Fig. 11b), compared 


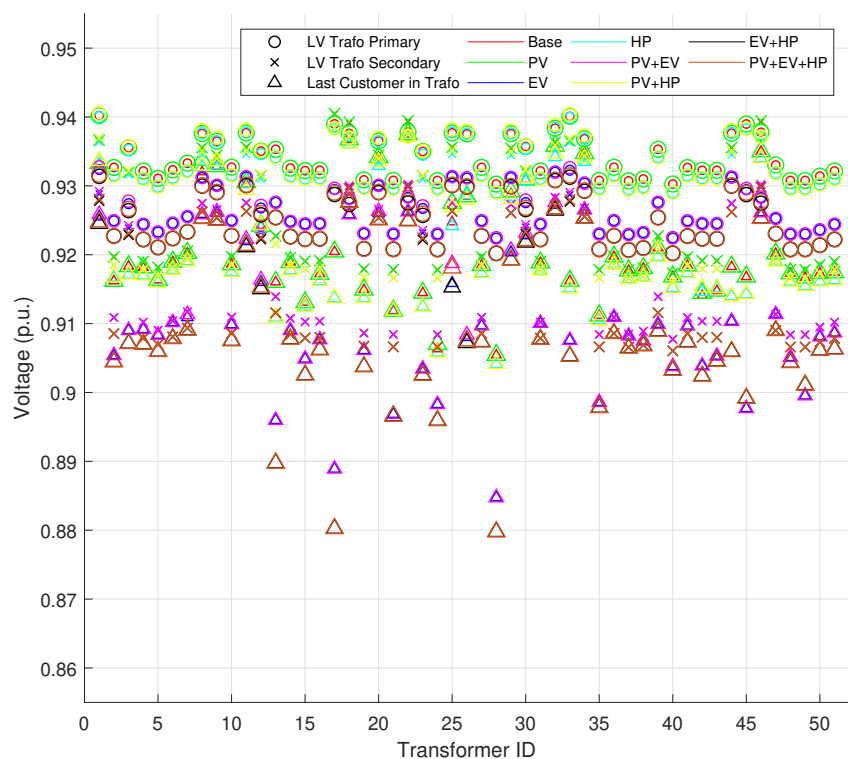

(a)

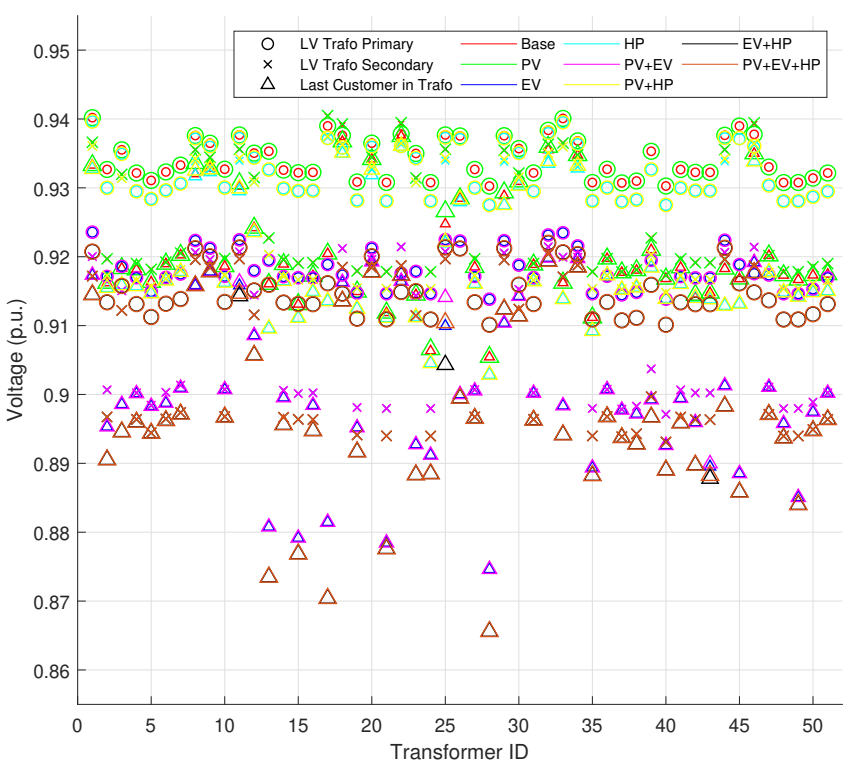

(b)

Fig. 9: Scatter plot containing the critical voltage of the day for each transformer, technology and measuting point (a) 10\% penetration (b) $15 \%$ penetration

to the sole EV integration case. On the other hand, it does not improve the lowest observed voltages since these voltages occur in the evening when there is no generation from PV. Combined integration of PV and HP helps increase the average voltages considerably compared to the sole HP integration case, while the highest and lowest observed voltages remain almost the same (see Fig. 11c). Combined integration of EV and HP results in slightly further decrease in the average and lowest observed voltages since their high demand times mostly do not coincide with each other (see Fig. 11d). The impacts for this case are particularly evident at the LV customer nodes. Addition of PV to EV and HP integration increases the average and the highest observed voltages, without considerable improvement in the lowest observed voltages and undervoltage risk as seen in Fig. 11e.

The analysis provided valuable findings for PV, EV and HP integration options into distribution networks. Due to higher per customer installed power and coincidence of EV and HP demand with residential peaks compared to lower coincidence of PV peaks with low demand times, undervoltage problems is primarily observed rather than overvoltage issues for the same penetration rates of technologies. Furthermore, it is possible to integrate EV and HP together with no considerable escalation of unintended impacts compared to sole EV integration, due to low coincidence of their daily peaks. Moreover, PV can be integrated together with EV, HP or both to help improving the overall voltage profiles; but not reducing the undervoltage risks considerably. Combined integration of PV with EV, HP or both is also helpful at reducing the risk of overvoltages that may be faced in uneven penetration rates (with high penetration of PV and relatively lower penetration of EV, HP or both) of technologies. The analysis highlighted the need to implement active mitigation methods especially to prevent undervoltage problems and increase hosting capacity of EV and HP.

\section{CONCLUSION}

This study presented a large scale MV/LV integrated distribution network modelling approach and comparative PV, EV, and HP integration analysis, considering combined integration scenarios. Different types of data from diverse databases were used to develop models of LV feeders in detail and conduct $\mathrm{MV} / \mathrm{LV}$ integrated quasi-static time series analysis at large scale, in collaboration with the DSO ESB Networks, in a pilot area in Ireland. The modelling study highlighted the challenges that DSO may face in further extended LV modelling efforts. The comparative analysis highlighted the suitable integration combinations of new technologies into existing networks. In combined integration with similar penetration levels, undervoltage problems are more likely to be faced rather than overvoltage issues. PV is useful to increase average voltage and the highest observed voltages in combined integration, while it does not prevent undervoltage events that occur in the evening or early in the morning, at times when supply from PV is not available. HP peak demand does not coincide with the daily residential peak, causing slight negative impacts compared to the base case. EV integration has considerable impacts on the average, highest and the lowest voltages, leading to undervoltage issues. Combined integration of EV and HP provided results similar to sole EV integration case, since their daily peak times (early morning for HP and late night for EV) do not coincide with each other. Addition of $\mathrm{PV}$ to EV and HP integration is useful to improve voltage profiles. However, active voltage mitigation techniques need to be implemented to prevent undervoltage and enable higher penetration rates of electrified transport and heat. The MV-LV area modelled has known challenges and plans are in place to upgrade the MV network from the existing $10 \mathrm{kV}$ network to $20 \mathrm{kV}$. Yet, the insights gained during the model development can assist in facilitating country-scale implementation. 
The future work will be on active management methods to mitigate the operational problems faced in integration of PV, $\mathrm{EV}$ and $\mathrm{HP}$ and analysis of the additional hosting capacities that can may become available in LV networks.

\section{ACKNOWLEDGMENTS}

The authors would like to thank Dr. Alison O'Connell of EPRI for her work in converting and validating the original MV model into OpenDSS.

\section{REFERENCES}

[1] L. Kristov, "The bottom-up (r)evolution of the electric power system: The pathway to the integrated-decentralized system," IEEE Power and Energy Magazine, vol. 17, no. 2, pp. 42-49, 2019.

[2] E. Hillberg, A. Zegers, B. Herndler, S. Wong, J. Pompee, J.-Y. Bourmaud, S. Lehnhoff, G. Migliavacca, K. Uhlen, I. Oleinikova et al., "Flexibility needs in the future power system," 2019.

[3] IEA. (2019) Global ev outlook 2019: Scaling up the transition to electric mobility. Accessed: 2022-01-25. [Online]. Available: http://webstore.iea.org/global-ev-outlook-2019

[4] International Energy Agency. (2022) Electric cars fend off supply challenges to more than double global sales. Accessed: 2022-02-07. [Online]. Available: https://www.iea.org/commentaries/ electric-cars-fend-off-supply-challenges-to-more-than-double-global-sale

[5] IEA. (2019) Renewables 2019 - market analysis and forecast from 2019 to 2024. Accessed: 2022-01-25. [Online]. Available: https://www.iea.org/reports/renewables-2019

[6] T. Stetz, M. Kraiczy, K. Diwold, M. Braun, B. Bletterie, C. Mayr R. Bründlinger, B. Noone, A. Bruce, I. MacGill et al., "High penetration pv in local distribution grids-outcomes of the iea pvps task 14 subtask 2,' in 29th European Photovoltaic Solar Energy Conference and Exhibition, vol. 15, no. 1, 2014, pp. 3994-3999.

[7] ESB Networks. (2020) Our infrastructure. Accessed: 2022-01-25. [Online]. Available: https://www.esbnetworks.ie/who-we-are/our-networks

[8] J. Peppanen, C. Rocha, J. A. Taylor, and R. C. Dugan, "Secondary lowvoltage circuit models-how good is good enough?" IEEE Transactions on Industry Applications, vol. 54, no. 1, pp. 150-159, 2017.

[9] M. A. Zehir, A. Batman, M. A. Sonmez, A. Font, D. Tsiamitros, D. Stimoniaris, T. Kollatou, M. Bagriyanik, A. Ozdemir, and E. Dialynas, "Impacts of microgrids with renewables on secondary distribution networks," Applied energy, vol. 201, pp. 308-319, 2017.

[10] A. Traupmann and T. Kienberger, "Test grids for the integration of res-a contribution for the european context," Energies, vol. 13, no. 20, p. 5431,2020 .

[11] C. Mateo, G. Prettico, T. Gómez, R. Cossent, F. Gangale, P. Frías, and G. Fulli, "European representative electricity distribution networks," International Journal of Electrical Power \& Energy Systems, vol. 99, pp. 273-280, 2018.

[12] K. Strunz, R. Fletcher, R. Campbell, and F. Gao, "Developing benchmark models for low-voltage distribution feeders," in 2009 IEEE Power \& Energy Society General Meeting. IEEE, 2009, pp. 1-3.

[13] S. Papathanassiou, N. Hatziargyriou, K. Strunz et al., "A benchmark low voltage microgrid network," in Proceedings of the CIGRE symposium: power systems with dispersed generation. CIGRE, 2005, pp. 1-8.

[14] CIGRE Task Force C6.04. (2014) Benchmark systems for network inte- gration of renewable and distributed energy resources. Accessed: 2022-01-25. [Online]. Available: https://e-cigre.org/publication/ELT 273_8-benchmark-systems-for-network-integration-of-renewable-and_ distributed-energy-resources

[15] J. Dickert, M. Domagk, and P. Schegner, "Benchmark low voltage distribution networks based on cluster analysis of actual grid properties," in 2013 IEEE Grenoble Conference. IEEE, 2013, pp. 1-6.

[16] M. Nijhuis, M. Gibescu, and J. Cobben, "Clustering of low voltage feeders from a network planning perspective," in Proceedings of the 23rd International Conference on Electricity Distribution (CIRED), 15 18 June 2015, Lyon, France, 2015, pp. 0680-1.

[17] V. Rigoni, L. F. Ochoa, G. Chicco, A. Navarro-Espinosa, and T. Gozel, "Representative residential lv feeders: A case study for the north west of england," IEEE Transactions on Power Systems, vol. 31, no. 1, pp. 348-360, 2015.
[18] V. Krishnan, B. Bugbee, T. Elgindy, C. Mateo, P. Duenas, F. Postigo, J.S. Lacroix, T. G. San Roman, and B. Palmintier, "Validation of synthetic us electric power distribution system data sets," IEEE Transactions on Smart Grid, vol. 11, no. 5, pp. 4477-4489, 2020.

[19] F. E. Postigo Marcos, C. Mateo Domingo, T. Gomez San Roman, B. Palmintier, B.-M. Hodge, V. Krishnan, F. de Cuadra García, and B. Mather, "A review of power distribution test feeders in the united states and the need for synthetic representative networks," Energies, vol. 10 , no. 11 , p. $1896,2017$.

[20] M. Deakin, D. Greenwood, S. Walker, and P. C. Taylor, "Hybrid european mv-lv network models for smart distribution network modelling," in 2021 IEEE Madrid PowerTech. IEEE, 2021, pp. 1-6.

[21] A. Koirala, L. Suárez-Ramón, B. Mohamed, and P. Arboleya, "Nonsynthetic european low voltage test system," International Journal of Electrical Power \& Energy Systems, vol. 118, p. 105712, 2020.

[22] G. Valverde, A. Arguello, R. González, and J. Quirós-Tortós, "Integration of open source tools for studying large-scale distribution networks," IET Generation, Transmission \& Distribution, vol. 11, no. 12, pp. 31063114, 2017.

[23] T. J. Morrell, V. Venkataramanan, A. Srivastava, A. Bose, and C.C. Liu, "Modeling of electric distribution feeder using smart meter data," in 2018 IEEE/PES Transmission and Distribution Conference and Exposition (T\&D). IEEE, 2018, pp. 1-9.

[24] K. Montano-Martinez, S. Thakar, S. Ma, Z. Soltani, V. Vittal, M. Khorsand, R. Ayyanar, C. Rojas et al., "Detailed primary and secondary distribution system model enhancement using ami data," arXiv preprint arXiv:2105.14161, 2021.

[25] J. See, W. Carr, and S. E. Collier, "Real time distribution analysis for electric utilities," in 2008 IEEE Rural Electric Power Conference. IEEE, 2008, pp. B5-B5.

[26] J. D. See, S. Latham, G. Shirek, and W. C. Carr, "Report on realtime grid analysis pilots," IEEE Transactions on Industry Applications, vol. 48, no. 4, pp. 1170-1176, 2012.

[27] M. J. Reno, J. Deboever, and B. Mather, "Motivation and requirements for quasi-static time series (qsts) for distribution system analysis," in 2017 IEEE Power \& Energy Society General Meeting. IEEE, 2017, pp. 1-5.

[28] I. Richardson, M. Thomson, D. Infield, and C. Clifford, "Domestic electricity use: A high-resolution energy demand model," Energy and buildings, vol. 42, no. 10, pp. 1878-1887, 2010.

[29] ESB Networks. (2021) Distribution system security and planning standards. Accessed: 2022-01-25. [Online]. Available: https://www.esbnetworks.ie/docs/default-source/publications/ distribution-system-security-and-planning-standards.pdf?sfvrsn= beedd2e5_23

[30] M. U. Qureshi, S. Grijalva, M. J. Reno, J. Deboever, X. Zhang, and R. J. Broderick, "A fast scalable quasi-static time series analysis method for pv impact studies using linear sensitivity model," IEEE Transactions on Sustainable Energy, vol. 10, no. 1, pp. 301-310, 2018.

[31] ESB Networks. (2021) The dingle project. Accessed: 2022 01-25. [Online]. Available: https://www.esbnetworks.ie/who-we-are/ innovation/esb-networks' -dingle-project

[32] I. Richardson and M. Thomson, "Integrated simulation of photovoltaic micro-generation and domestic electricity demand: a one-minute resolution open-source model," proceedings of the institution of Mechanical Engineers, Part A: Journal of Power and Energy, vol. 227, no. 1, pp. 73-81, 2013.

[33] F.-L. Heng, K. Zhang, A. Goyal, H. Chaudhary, S. Hirsch, Y. Kim, M. A. Lavin, and A. Raman, "Integrated analytics system for electric industry asset management," IBM Journal of Research and Development, vol. 60, no. 1, pp. 2-1, 2016.

[34] Commission for Regulation of Utilities. (2020) Distribution system operator (dso) revenue for 2021-2025. Accessed: 2022-0125. [Online]. Available: https://www.cru.ie/wp-content/uploads/2020/07/ CRU20077-Distribution-System-Operator-DSO-Revenue-for-2021-2025. pdf

\section{APPENDIX \\ Detailed Comparisons of CASE Studies}

Voltage measured at different points in the base case and the cases and its combinations with $15 \%$ penetration level are shown in Fig. 10 and Fig. 11. 


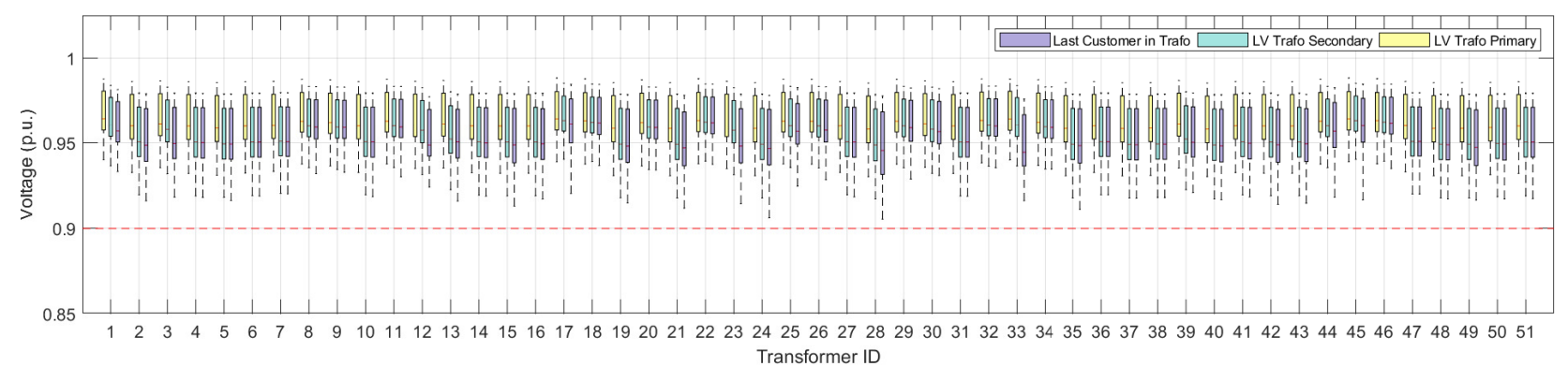

(a)

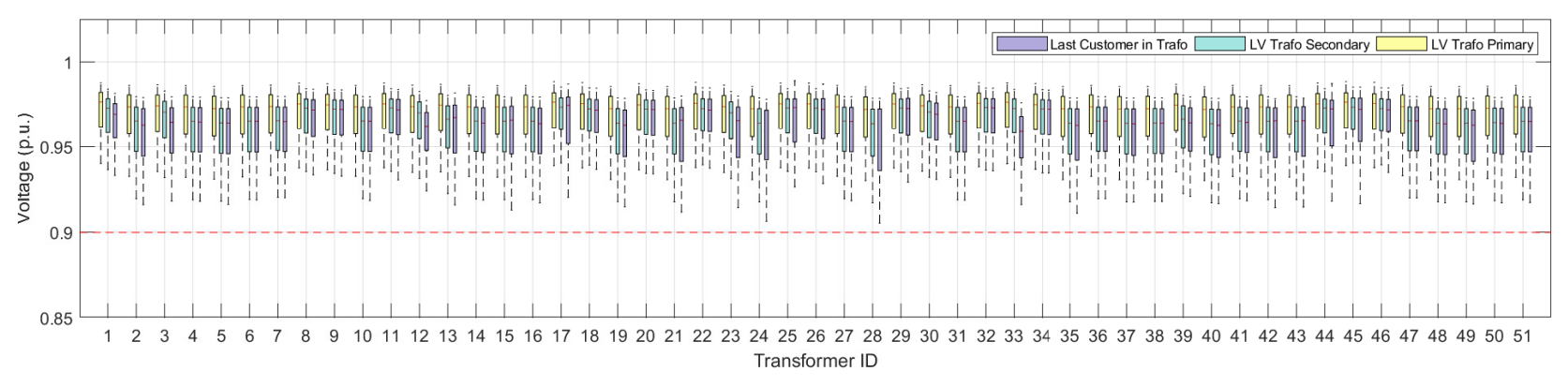

(b)

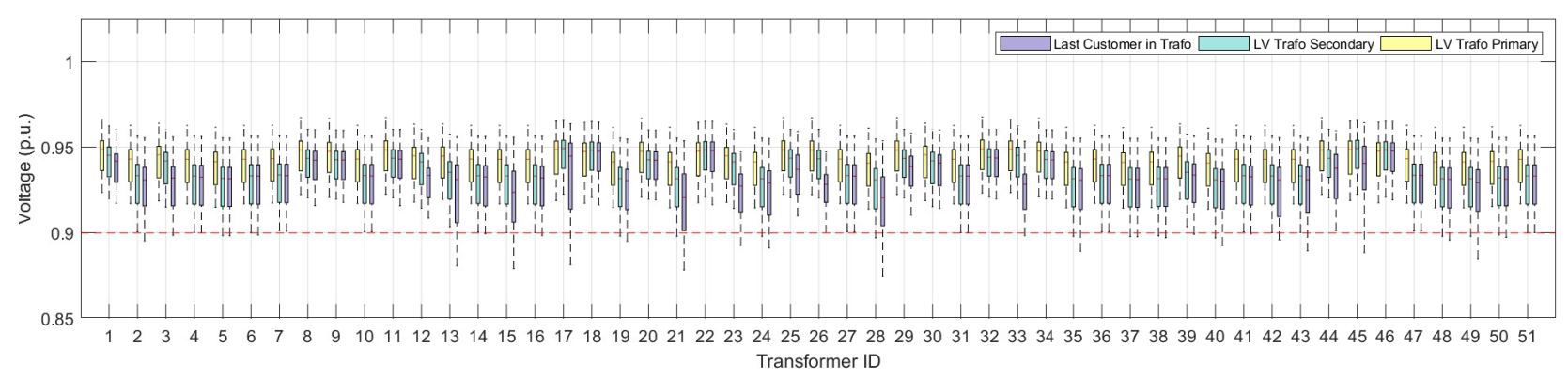

(c)

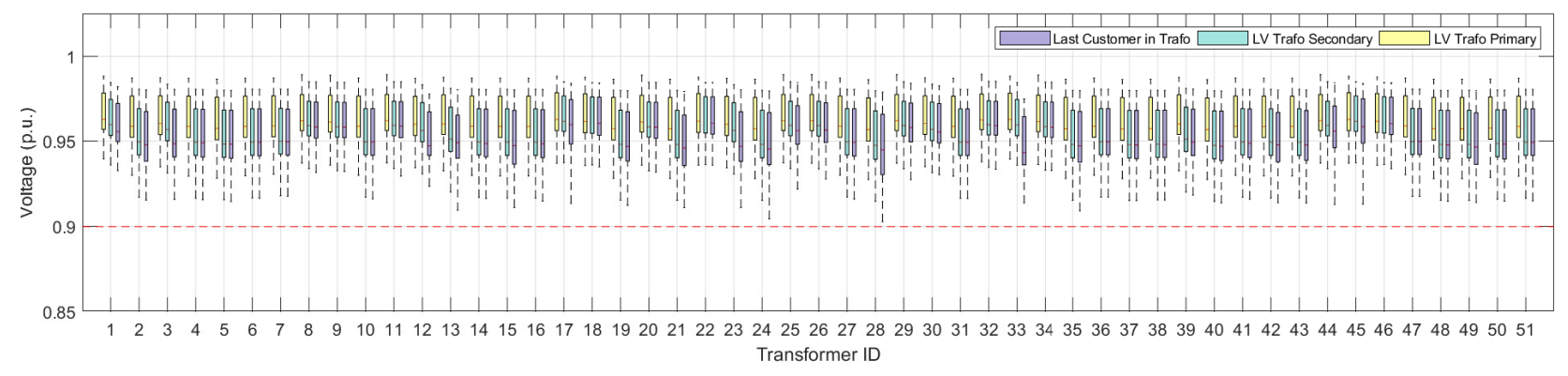

(d)

Fig. 10: Voltage measured at different points in the base case and the individual cases with $15 \%$ penetration level (a) Base Case (b) PV only (c) EV only (d) HP only. 


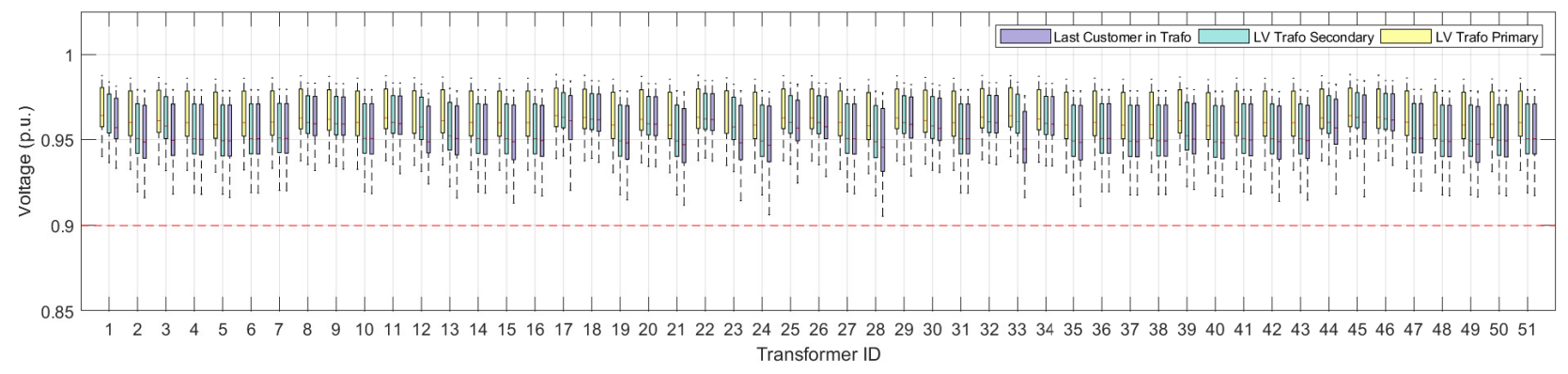

(a)

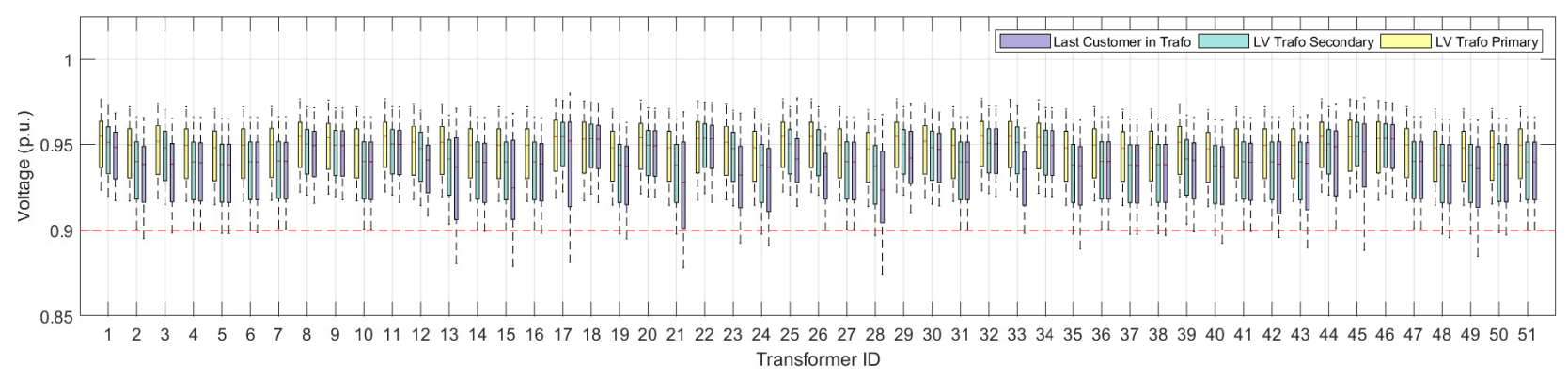

(b)

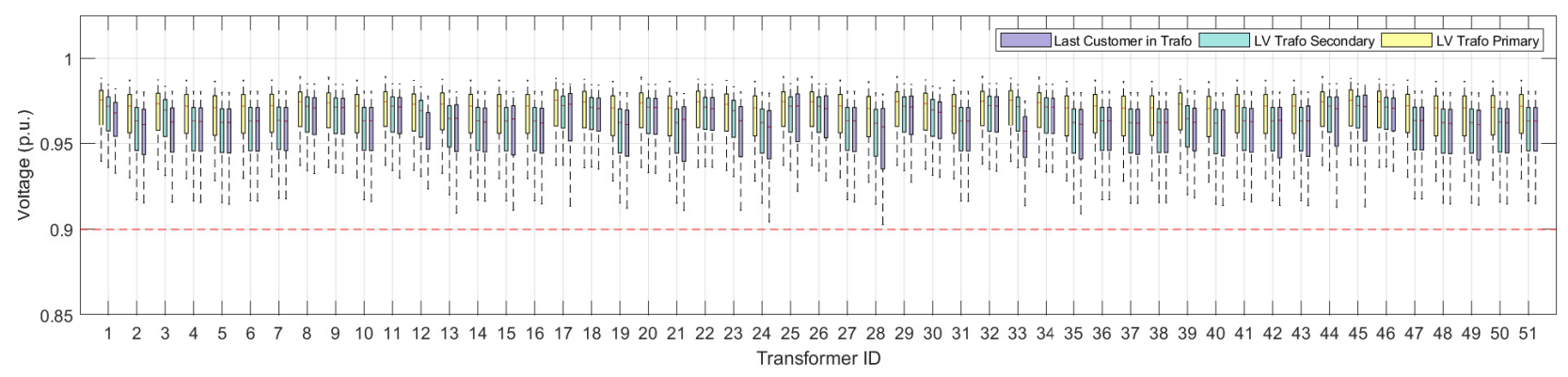

(c)

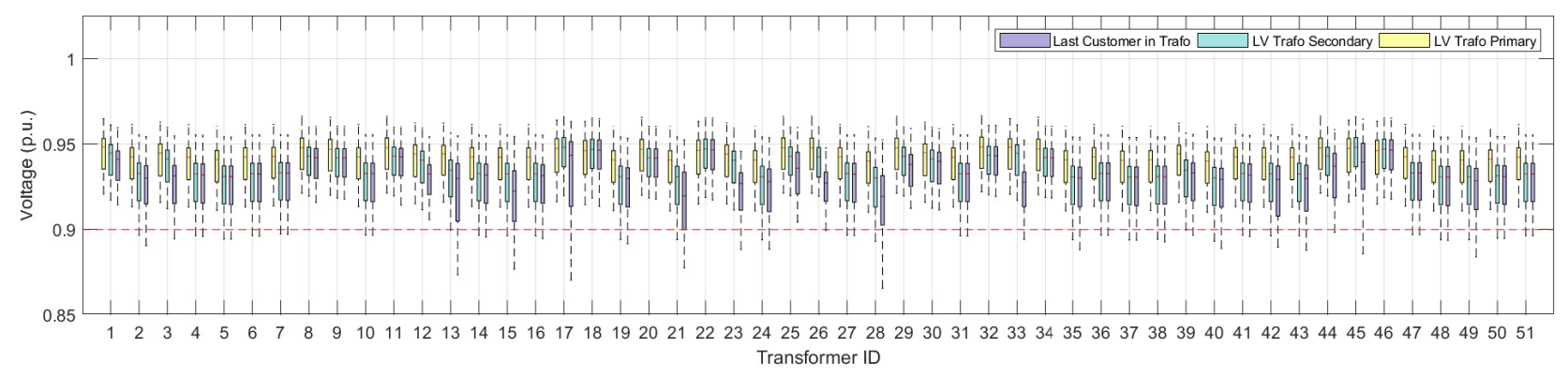

(d)

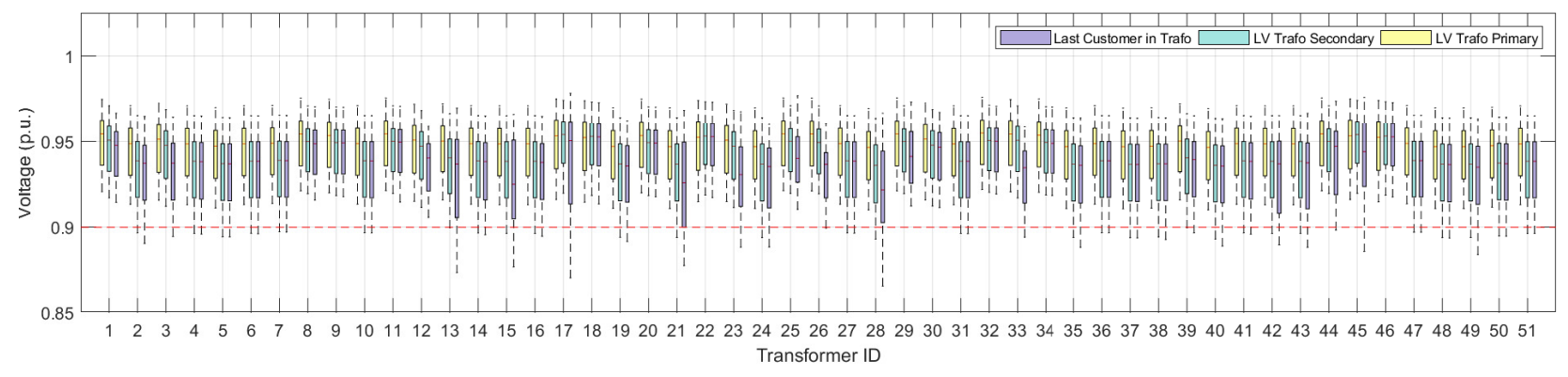

(e)

Fig. 11: Voltage measured at different points in the base case and the combined cases with $15 \%$ penetration level (a) Base Case (b) PV and EV Case (c) PV and HP Case (d) EV and HP Case (e) PV, EV and HP Case 